\title{
CRESCIMENTO E TEOR DE METAIS DE MUDAS DE ESPÉCIES ARBÓREAS CULTIVADAS EM SOLO CONTAMINADO COM METAIS PESADOS ${ }^{1}$
}

\author{
TERESA CRISTINA LARA LANZA DE SÁE MELO MARQUES², \\ FATIMA MARIA DE SOUZA MOREIRA ${ }^{3}$ e JOSÉ OSWALDO SIQUEIRA ${ }^{3}$
}

\begin{abstract}
RESUMO - O objetivo do trabalho foi avaliar o teor de metais pesados e o crescimento de mudas de 20 espécies arbóreas tropicais em solo com elevado grau de contaminação com metais pesados. Em casa de vegetação, as mudas foram transplantadas para vasos contendo $3,3 \mathrm{~kg}$ de misturas com diferentes proporções $(0,20,40$ e $60 \% \mathrm{v} / \mathrm{v})$ de solo contaminado. Verificou-se comportamento diferenciado das espécies quanto à inibição de crescimento e aos teores de metais na raiz e na parte aérea em decorrência da contaminação. Com base na produção de matéria seca da parte aérea, concluiu-se que apenas Myrsine umbellata, Cedrella fissilis, Tabebuia impetiginosa e Copaifera langsdorffii não foram afetadas pela contaminação, enquanto Hymenaea courbaril, Mimosa caesalpiniaefolia, Acacia mangium e Platypodium gonoacantha sofreram pequeno impacto. As demais espécies foram muito inibidas pela contaminação do solo, o que é causado pela absorção, na maioria dos casos, de Zn e Cd. Várias espécies apresentaram elevada capacidade de reter esses metais nas raízes, evitando sua translocação para a parte aérea.
\end{abstract}

Termos para indexação: poluição do solo, árvores tropicais, revegetação, reabilitação do solo.

\section{GROWTH AND METAL CONCENTRATION OF SEEDLINGS OF WOODY SPECIES IN A HEAVY METAL CONTAMINATED SOIL}

\begin{abstract}
The objective of the paper was to evaluate metal content and ability to grow in soil with excess of heavy metals of seedlings of 20 woody species. In the greenhouse, seedlings were transplanted to pots with $3.3 \mathrm{~kg}$ of soil-mixes with different proportions $(0,20,40,60 \% \mathrm{v} / \mathrm{v})$ of a heavy metal contaminated soil. It was found that plant species behaved differently in terms of growth inhibition and metal content in the shoots and roots. Based upon the dry matter yield, only Myrsine umbellata, Cedrella fissilis, Tabebuia impetiginosa and Copaifera langsdorffii were not affected by increasing contamination, whereas Hymenaea courbaril, Mimosa caesalpiniaefolia, Acacia mangium and Platypodium gonoacantha were only slightly affected by it. All the other species were highly inhibited by the excess of metals in the soil, being such effects related, in most cases, to high uptake of $\mathrm{Zn}$ and $\mathrm{Cd}$. Several species were able to retain large quantities of these metals in the roots, therefore avoiding their translocation to shoots.
\end{abstract}

Index terms: soil pollution, tropical trees, revegetation, soil rehabilitation.

\section{INTRODUÇÃO}

Ao mesmo tempo que a contaminação com metais pesados afeta o crescimento, a distribuição e o

\footnotetext{
${ }^{1}$ Aceito para publicação em 25 de fevereiro de 1999 Trabalho financiado pelo Convênio FAEPE/CMM e FAPEMIG.

${ }^{2}$ Eng. Agrôn., M.Sc., 149-11 Arnold drive, West Lafayette IN, 47906-3339, EUA. E-mail: tmarques@expert.cc.purdue.edu

${ }^{3}$ Eng. Agrôn., Ph.D., Dep. de Ciência do Solo, Universidade Federal de Lavras, Caixa Postal 37, CEP 37200-000 Lavras, MG. Bolsista do CNPq.
}

ciclo biológico das espécies vegetais (Barceló \& Poschenrieder, 1992), a vegetação é uma alternativa para a recuperação de solos degradados pelo excesso desses elementos (Salt et al., 1995), devendo as espécies vegetais ser avaliadas quanto a sua capacidade de tolerância a esse tipo de estresse. Apesar de os mecanismos de tolerância a metais pesados serem pouco conhecidos, sabe-se que as espécies tolerantes não diferem morfologicamente das não-tolerantes ou sensíveis aos metais, mas diferem bioquímica e fisiologicamente. Segundo Baker (1987), a sobrevivência das espécies que crescem em solos 
contaminados é relacionada à capacidade destas de tolerar, e não de anular, a toxicidade do metal.

A concentração e o acúmulo de metais nos tecidos da planta dependem de sua disponibilidade na solução do solo, pois a concentração desses na raiz e na parte aérea aumenta com o aumento da sua concentração na solução do solo. As espécies tolerantes geralmente acumulam maiores concentrações de metais pesados na raiz em relação à parte aérea (Baker, 1987; Verkleij \& Prast, 1989). Isso indica que as plantas que crescem nessas condições não conseguem evitar a absorção dos metais, mas limitam sua translocação. Em populações de Silene sp., por exemplo, Verkleij \& Prast (1989) verificaram que a retenção de $\mathrm{Cd}$ na raiz forma a base de tolerância dessas ao excesso deste elemento. Espécies tolerantes podem ser caracterizadas de acordo com a capacidade relativa em absorver, translocar e concentrar os metais na planta, pois as plantas são consideradas acumuladoras, indicadoras e excluidoras, de acordo com as concentrações relativas dos metais na raiz e na parte aérea (Baker, 1981). Casos há em que as plantas têm mais de $100 \mathrm{mg} \mathrm{kg}^{-1} \mathrm{de} \mathrm{Cd}, 1.000 \mathrm{mg} \mathrm{kg}^{-1}$ de $\mathrm{Ni}$ e $\mathrm{Cu}$ ou $10.000 \mathrm{mg} \mathrm{kg}^{-1}$ de $\mathrm{Zn}$ na matéria seca, quando crescem em solos ricos nestes metais, sendo chamadas de hiperacumuladoras (Brown et al., 1995).

Os níveis tóxicos dos metais pesados para as plantas são ainda pouco conhecidos. Concentrações de alguns metais pesados consideradas tóxicas no tecido vegetal têm sido sugeridas (Kabata-Pendias \& Pendias, 1984), porém esses valores são muito amplos, variáveis e totalmente desconhecidos para plantas não-cultivadas, como as arbóreas tropicais. Essas, pela capacidade de imobilizar maior quantidade de metais nos tecidos em relação às herbáceas, apresentam grande potencial para programas de revegetação de áreas contaminadas com metais pesados.

No presente estudo avaliaram-se os teores de metais pesados e suas relações com o crescimento de mudas de 20 espécies arbóreas em mistura de solo, contendo diferentes proporções de um solo com elevado grau de contaminação por diversos metais pesados.

\section{MATERIAL E MÉTODOS}

O experimento foi desenvolvido com mudas formadas em viveiro e transplantadas para vasos em casa de vegetação do Departamento de Ciência do Solo da Universidade Federal de Lavras (UFLA), no período de janeiro a abril de 1996. Utilizaram-se amostras de um solo contaminado por metais pesados, coletado em área de rejeito, na Companhia Mineira de Metais (CMM), no município de Três Marias, MG, aqui denominado solo CMM, e de um Latossolo Vermelho-Escuro (LE) não- contaminado, coletado na camada superficial $(0-20 \mathrm{~cm})$, no município de São Sebastião da Vitória, MG. Em face do elevado grau de contaminação do solo CMM (teores totais em $\mathrm{mg} \mathrm{kg}^{-1}$ de solo: $\mathrm{Zn}=18.600 ; \mathrm{Cd}=135 ; \mathrm{Pb}=600$ e $\mathrm{Cu}=596$, além de outros em menores teores), este foi diluído em diferentes proporções com o LE não- contaminado, de modo a obter as seguintes proporções: $0,20,40$ e $60 \%(\mathrm{v} / \mathrm{v})$ de solo contaminado. Ambos os solos foram secados ao ar e passados em peneira de malha $5 \mathrm{~mm}$. Com base na análise química do material de solo, procedeu-se à correção de acidez com $\mathrm{Ca}(\mathrm{OH})_{2}$ puro e do nível de $\mathrm{P}$ do solo como superfosfato triplo ( $200 \mathrm{mg} \mathrm{kg}^{-1} \mathrm{de} \mathrm{P}$ ), de forma a elevar a saturação por base e nível de $\mathrm{P}$ do solo diluente (LE) a valores próximos ao solo contaminado, antes da mistura. O LE foi incubado por 15 dias, com o teor de umidade em torno de $70 \%$ do volume total de poros (VTP). Passado esse período, prepararam-se as diferentes misturas de solo, as quais receberam vermicomposto $(10 \%$ por peso úmido). As misturas foram completamente homogeneizadas e colocadas em vasos de plástico contendo $3,3 \mathrm{~kg}$ de solo. Amostras foram retiradas de cada proporção de solo contaminado, para análise química (Tabela 1). As análises de fertilidade foram realizadas, conforme Embrapa (1979), nos laboratórios da UFLA, e os teores totais dos metais pesados no solo no laboratório da CMM em Três Marias, MG, após digestão com água régia conforme Foster (1995), e determinação por espectrofotometria de absorção atômica.

$\mathrm{O}$ delineamento experimental foi inteiramente casualizado, com cinco repetições, em esquema fatorial $20 \times 4$, sendo 20 espécies arbóreas (Tabela 2) e quatro diferentes proporções de solo contaminado. As espécies foram selecionadas considerando-se o seu potencial para programas de recomposição florística de áreas degradadas, e as proporções de solo contaminado foram determinadas em ensaios prévios realizados neste departamento. O plantio foi realizado empregando-se mudas com raízes seminuas, com desenvolvimento adequado para o plantio no campo. A idade das plantas variou de 6 a 13 meses, e a altura, de 5 a $95 \mathrm{~cm}$, dependendo da espécie. As mudas foram formadas em viveiro, em sacos de plástico, em substrato com- 
posto de terra de subsolo misturado com palha de arroz carbonizada, sendo transplantada uma muda por vaso. No transplante, o substrato aderido à muda foi eliminado por meio de lavagens, para reduzir a interferência na resposta das plantas à contaminação da mistura de solo. O experimento foi conduzido por 90 dias, em casa de vegetação, mantendo-se o solo com umidade próxima de $60 \%$ do VTP. Aos $15,45,75$ e 90 dias após o transplantio das mudas, foram avaliadas as alturas das plantas, e por meio delas determinou-se o ganho relativo após 45, 75 e 90 dias. Este foi calculado pela razão das diferenças de altura em cada época, e a altura, aos 15 dias após transplantio entre solo contaminado e solo controle, multiplicado por 100. Aos 90 dias após transplantio, as plantas foram colhidas e separadas em raiz e parte aérea, secas em estufa com circulação de ar a $70^{\circ} \mathrm{C}$, pesadas e moídas. Posteriormente, efetuou-se a digestão nitroperclórica do material para determinação, nos extratos, de $\mathrm{Cu}, \mathrm{Cd}, \mathrm{Fe}, \mathrm{Mn}$ e $\mathrm{Pb}$ por espectrofotometria de absorção atômica. Em vista dos elevados teores de $\mathrm{Zn}$ na matéria seca, e da necessidade de diluições, optou-se pelas leituras dos teores deste elemento por espectrometria de emissão atômica com plasma,

TABELA 1. Características químicas das misturas com diferentes proporções de solos contaminados.

\begin{tabular}{|c|c|c|c|c|c|c|c|c|c|c|c|c|c|c|}
\hline $\begin{array}{l}\text { Proporção de solo } \\
\text { contaminado }\end{array}$ & $\begin{array}{c}\mathrm{pH} \\
\text { (água) }\end{array}$ & $\begin{array}{l}\text { Mat. } \\
\text { org. }\end{array}$ & $\mathrm{P}$ & $\mathrm{K}$ & $\mathrm{Ca}$ & $\mathrm{Mg}$ & $\mathrm{H}+\mathrm{Al}$ & $\mathrm{Al}$ & $\mathrm{Fe}$ & $\mathrm{Cd}$ & $\mathrm{Cu}$ & $\mathrm{Zn}$ & $\mathrm{Mn}$ & $\mathrm{Pb}$ \\
\hline$(\%)$ & & $\left(\mathrm{g} \mathrm{kg}^{-1}\right)$ & \multicolumn{2}{|c|}{$-\left(\mathrm{mg} \mathrm{kg}^{-1}\right)-$} & \multicolumn{4}{|c|}{------- $\left(\mathrm{mmol}_{\mathrm{c}} \mathrm{dm}^{-3}\right)$------ } & \multicolumn{6}{|c|}{-------------------- (mg kg } \\
\hline 0 & 6,6 & 43 & 27 & 120 & 31 & 17 & 26 & 1 & 73.200 & 2 & 30 & 76 & 100 & 20 \\
\hline 20 & 6,4 & 40 & 37 & 128 & 28 & 20 & 23 & 1 & 68.900 & 35 & 390 & 3.878 & 354 & 202 \\
\hline 40 & 6,3 & 35 & 43 & 103 & 26 & 19 & 21 & 1 & 59.500 & 75 & 1.000 & 8.800 & 586 & 427 \\
\hline 60 & 6,4 & 34 & 44 & 133 & 26 & 13 & 17 & 1 & 47.700 & 106 & 1.490 & 14.461 & 862 & 707 \\
\hline
\end{tabular}

TABELA 2. Espécies arbóreas estudadas e altura média das mudas por ocasião do plantio nos vasos.

\begin{tabular}{lllc}
\hline Nome comum & Nome científico & Família & $\begin{array}{c}\text { Altura } \\
(\mathrm{cm})\end{array}$ \\
\hline Acácia & Acacia mangium L. & Mimosaceae & 16,6 \\
Angico-amarelo & Peltophorum dubium (Spreng) Taub. & Caesalpiniaceae & 16,2 \\
Angico-vermelho & Anadenanthera peregrina (Benth.) Speg. & Mimosaceae & 26,2 \\
Bico-de-pato & Machaerium nictidans Benth. & Fabaceae & 28,8 \\
Cedro & Cedrella fissilis Vell. & Meliaceae & 22,3 \\
Espeto & Casearia lasiophylla Eichler & Flacourtiaceae & 37,7 \\
Fedegoso & Senna macranthera (Vell.) I \& B. & Caesalpiniaceae & 20,2 \\
Gravitinga & Solanum granulosum-leprosum Dun. & Solanaceae & 14,6 \\
Ipê-roxo & Tabebuia impetiginosa (Mart.) Standl. & Bignoniaceae & 9,5 \\
Jacarandá-branco & Platypodium gonoacantha (Mart.) Macbr. & Fabaceae & 26,4 \\
Jacaré & Piptadenia gonoacantha (Mart.) Macbr. & Mimosaceae & 12,7 \\
Jatobá & Hymenaea courbaril L. & Caesalpiniaceae & 45,9 \\
Louro & Cordia trichotoma (Vell.) Arrab. & Boraginaceae & 29,1 \\
Maria mole & Dendropanax cuneatum (DC.) Dcne. \& Planch & Araliaceae & 43,4 \\
Óleo-bálsamo & Myroxylon peruiferum L. & Fabaceae & 19,3 \\
Óleo-copaíba & Copaifera langsdorffii Desf. & Caesalpiniaceae & 13,2 \\
Pororoca & Myrsine umbellata Mart. & Myrsinaceae & 24,9 \\
Sabiá & Mimosa caesalpiniaefolia Benth. & Mimosaceae & 49,8 \\
Sebastiana & Sebastiania schottiana & Euphorbiaceae & 82,4 \\
Trema & Trema micrantha Blume & Ulmaceae & 21,2 \\
\hline
\end{tabular}


induzido em argônio (ICP/AES). A partir dos teores de $\mathrm{Zn}, \mathrm{Cd}, \mathrm{Pb}, \mathrm{Cu}, \mathrm{Fe}$ e $\mathrm{Mn}$ na matéria seca da parte aérea e raízes das plantas, calculou-se a razão entre o teor do elemento na raiz e na parte aérea (R/PA), e o coeficiente de impacto da contaminação no teor relativo (CITR). Este índice expressa a razão entre R/PA na mistura com $60 \%$ de solo contaminado e a R/PA no solo sem contaminação.

Os dados foram submetidos à análise da variância, a testes de médias, pelo programa estatístico SANEST (Zonta et al., 1984), e a testes de regressão, pelo programa SAEG (Universidade Federal de Viçosa, 19--).

\section{RESULTADOS E DISCUSSÃO}

O crescimento em altura, das espécies, foi influenciado $(\mathrm{P} \leq 0,05)$ pela contaminação. Dadas as diferenças iniciais na altura das mudas e na velocidade de crescimento destas após o transplante, optou-se pelo ganho relativo na altura (Fig. 1) para avaliar os efeitos da contaminação no crescimento das plantas. Verificam-se diferenças de comportamento entre as espécies, proporção de solo contaminado e época de avaliação. A espécie M. peruiferum foi muito afetada em todas as proporções de solo contaminado e épocas de avaliação, fato também observado na Platypodium gonoacantha, que teve acentuada redução no ganho relativo em altura com o período de crescimento após o transplante, fato este não observado em outras espécies. A Piptadenia gonoacantha e a A. peregrina foram drasticamente afetadas no tratamento com $60 \%$ de solo contaminado em relação às demais proporções. Diversas espécies, como a M. umbellata, Piptadenia gonoacantha, T. micrantha, P. dubium, S. macranthera, H. courbaril, C. lasiophylla, D. cuneatum, $M$. niticdans, $M$. caesalpiniaefolia e C. trichotoma, foram pouco afetadas no tratamento com $20 \%$ de solo contaminado (ganho relativo $>50 \%$ ), porém muito sensíveis ao aumento da concentração de metais no solo, o que evidencia a baixa tolerância destas a níveis elevados dos poluentes. Com base nesse parâmetro, C. fissilis foi a espécie que melhor tolerou a contaminação do solo, uma vez que seu ganho relativo, no tratamento com $60 \%$ de solo contaminado, foi superior a $100 \%$. T. impetiginosa teve aumento no ganho relativo de altura em todos os tratamentos contendo solo contaminado durante os períodos avaliados. Outras como A. mangium, C. langsdorffii, S. granulosoleprosum e $S$. schottiana, apesar de terem seu crescimento em altura afetado pela contaminação, toleraram a elevação da proporção de solo contaminado na mistura.

A produção de matéria seca da parte aérea também foi muito influenciada pela contaminação do solo, sendo o impacto desse fator diferenciado entre as espécies (Fig. 2). Apenas M. umbellata, C. fissilis, T. impetiginosa e $C$. langsdorffii não tiveram a produção de matéria seca influenciada pela contaminação. Outras, como H. courbaril, M. caesalpiniaefolia, A. mangium e Platypodium gonoacantha, apesar de serem afetadas significativamente, sofreram pequeno impacto da contaminação. Portanto, com base na produção de matéria seca, diversas espécies exibiram alguma tolerância à contaminação do solo. Verificou-se que em $C$. fissilis houve pequeno estímulo à produção de massa de raízes, fato também observado para crescimento em altura (Fig. 1).

A contaminação elevou significativamente os teores de $\mathrm{Zn}$ na matéria seca da parte aérea e raízes de todas as espécies, exceto em $T$. impetiginosa, que teve baixos teores deste elemento na matéria seca da parte aérea (Tabela 3). Outras espécies, apesar da elevação significativa no teor de $\mathrm{Zn}$, em face da elevação na proporção de solo contaminado, apresentaram também baixos teores desse elemento na matéria seca da parte aérea. Observa-se grande variação nos teores de $\mathrm{Zn}$ nas espécies, sendo estes mais evidentes no tratamento com $60 \%$ de solo contaminado, onde os teores deste elemento variaram de 290 a $1.330 \mathrm{mg} \mathrm{kg}^{-1}$ de matéria seca da parte aérea. $\mathrm{O} \mathrm{Zn}$ é considerado um metal de fitotoxidez baixa a moderada; a maioria das espécies estudadas até o presente são afetadas por concentrações no tecido, que variam de 100 a $400 \mathrm{mg} \mathrm{kg}^{-1}$ (KabataPendias \& Pendias, 1984). Portanto, na mistura com $60 \%$ de solo contaminado, todas as espécies tiveram teores nessa faixa ou maiores. No entanto, o aumento da disponibilidade desse elemento no solo não resultou em grande elevação do teor na parte aérea de algumas espécies. Os teores de $\mathrm{Zn}$ nas raízes foram maiores que na parte aérea, atingindo valores de até $5.780 \mathrm{mg} \mathrm{kg}^{-1} \mathrm{em}$ A. mangium. Contudo, nenhuma espécie estudada neste trabalho pode ser con- 
siderada hiperacumuladora (Brown et al., 1995). No tratamento com $60 \%$ de solo contaminado, todas as espécies, exceto M. umbellata, acumularam proporcionalmente mais $\mathrm{Zn}$ nas raízes (Tabela 3), embora os valores da razão R/PA situem-se próximos da unidade em algumas espécies. Desse modo, conclui-se que a maioria das espécies possui capacidade de reter $\mathrm{Zn}$ nas raízes, evitando sua translocação para a parte aérea. De acordo com Antosiewicz (1992), essas espécies comportam-se como plantas indicadoras.

Os teores de Cd comportaram-se de modo semelhante ao que foi observado quanto ao $\mathrm{Zn}$, apesar de serem encontrados valores bem menores (Tabela 4). As plantas apresentaram teores de $\mathrm{Cd}$ menores que $1,0 \mathrm{mg} \mathrm{kg}^{-1}$ no solo não-contaminado, enquanto no
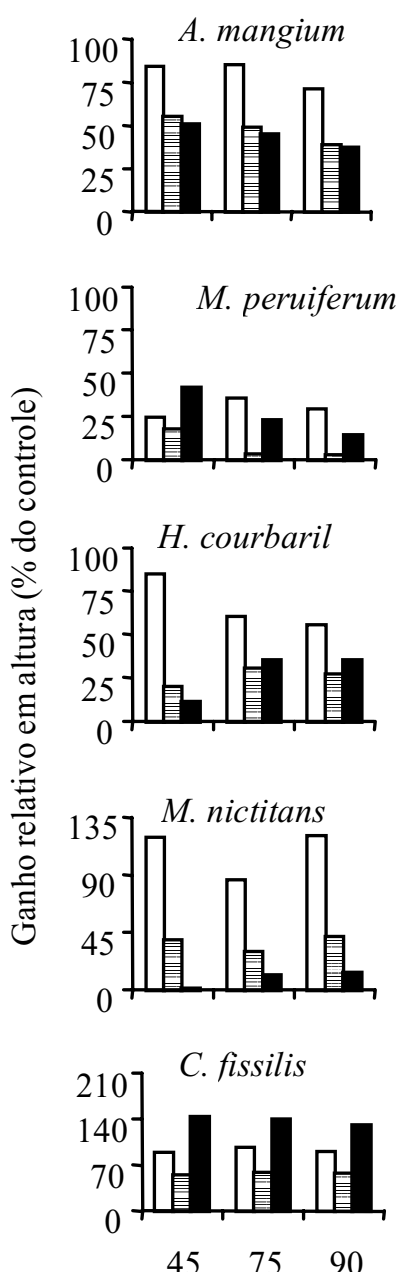
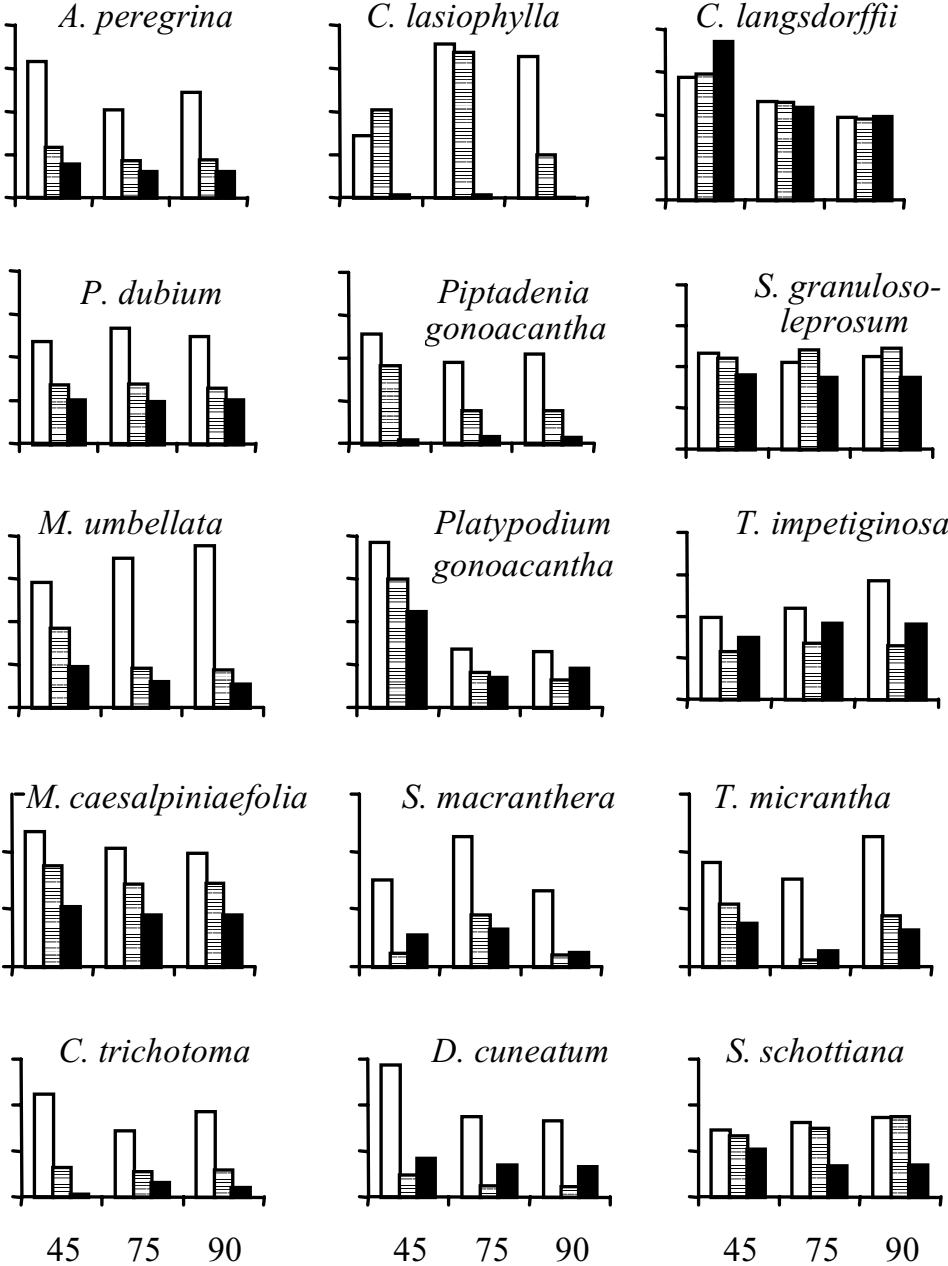

Dias após transplantio
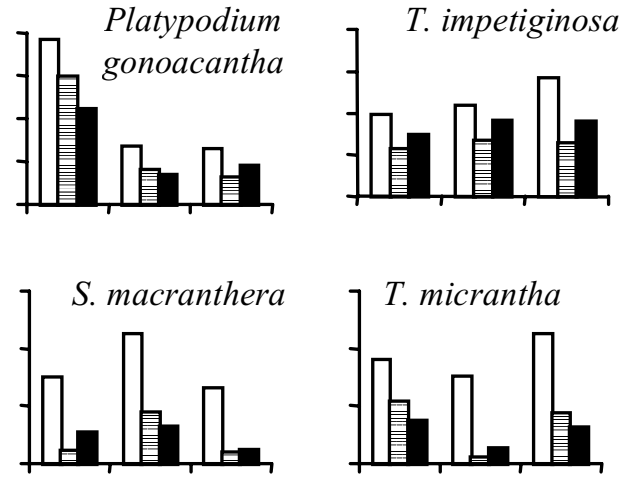

$\square 20 \% \quad$ 琶 $40 \% \square 60 \%$

FIG. 1. Ganho relativo em altura das espécies nos tratamentos com diferentes proporções de solo contaminado $(20 \%, 40 \%$ e $60 \%$ v/v) e dias após o transplantio (incremento no tratamento sem contaminação $=100 \%$ ). 
tratamento com $60 \%$ de solo contaminado a concentração desse elemento atingiu até $64 \mathrm{mg} \mathrm{kg}^{-1}$ de matéria seca. Como teores entre 5 e $30 \mathrm{mg} \mathrm{kg}^{-1}$ são considerados fitotóxicos (Kabata-Pendias \& Pendias, 1984), os resultados indicam que esse elemento foi também responsável pela redução de crescimento das espécies estudadas. Os teores de $\mathrm{Pb}$ foram ainda menores que os de $\mathrm{Cd}$. No tratamento com $60 \%$ de solo contaminado houve aumento nos teores de $\mathrm{Pb}$ em várias espécies (Tabela 5). Teores máximos foram $7,4 \mathrm{mg} \mathrm{kg}^{-1}$ na parte aérea de $A$. peregrina e $37,5 \mathrm{mg} \mathrm{kg}^{-1}$ nas raízes de T. impetiginosa. Considerando os níveis fitotóxicos de $\mathrm{Pb}$ (30 -300 mg kg-1) adotados por Kabata-Pendias \& Pendias (1984), esse elemento parece não ter sido problema nesse solo.

Ao contrário do que ocorreu nas raízes, algumas espécies como $C$. fissilis, H. courbaril, M. caesalpiniaefolia, S. schottiana, M. peruiferum,
M. umbellata e T. impetiginosa apresentaram proporcionalmente baixos teores de $\mathrm{Zn}$ e $\mathrm{Cd}$ na parte aérea, indicando algum mecanismo regulador da translocação desses metais da raiz para a parte aérea, o que pode ser uma estratégia de tolerância ao excesso de metais no solo (Antosiewicz, 1992). De fato, A. mangium, H. courbaril e M. caesalpiniaefolia estão entre as espécies cujo crescimento foi pouco afetado pela contaminação. A espécie $C$. fissilis, que não teve crescimento afetado pela contaminação apresentou resposta diferenciada quanto aos diferentes elementos contaminantes do solo, além de reduzida absorção e translocação desses da raiz para a parte aérea. Em geral, as espécies mais afetadas pela contaminação (Fig. 2) apresentaram pequena retenção proporcional de $\mathrm{Zn}$ e $\mathrm{Cd}$ nas raízes, os quais exerceram influência negativa em grande número de espécies (Tabela 6). As maiores inconsistências nos valores de R/PA foram

TABELA 3. Médias e regressão do teor de $\mathrm{Zn}$ na parte aérea e na raiz e razão $\mathrm{Zn}$ na raiz/Zn na parte aérea (R/PA) de mudas de espécies arbóreas, cultivadas em diferentes proporções de solo contaminado com metais pesados.

\begin{tabular}{|c|c|c|c|c|c|c|c|c|c|c|c|c|c|}
\hline \multirow[t]{2}{*}{ Espécie } & \multicolumn{4}{|c|}{$\begin{array}{l}\text { Proporção de solo } \\
\text { contaminado (\%) }\end{array}$} & \multicolumn{2}{|c|}{ Regressão $^{1}$} & \multicolumn{4}{|c|}{$\begin{array}{l}\text { Proporção de solo } \\
\text { contaminado }(\%)\end{array}$} & \multicolumn{2}{|c|}{ Regressão } & \multirow[t]{2}{*}{$\mathrm{R} / \mathrm{PA}^{2}$} \\
\hline & 0 & 20 & 40 & 60 & Mod. & $r^{2}$ & 0 & 20 & 40 & 60 & Mod. & $\mathrm{r}^{2}$ & \\
\hline & \multicolumn{6}{|c|}{--- Parte aérea $\left(\mathrm{mg} \mathrm{kg}^{-1}\right)$--- } & \multicolumn{6}{|c|}{----- Raiz $\left(\mathrm{mg} \mathrm{kg}^{-1}\right)$--.--- } & \\
\hline A. mangium & 60 & 260 & 640 & 760 & $\mathrm{~L}$ & $0,96 * *$ & 100 & 2.960 & 3.300 & 5.780 & $\mathrm{~L}$ & $0,93 * *$ & 8,0 \\
\hline A. peregrina & 30 & 170 & 320 & 630 & $\mathrm{~L}$ & $0,96 * *$ & 110 & 320 & 380 & 1.180 & $\mathrm{~L}$ & $0,81 * *$ & 1,9 \\
\hline C. lasiophylla & 60 & 250 & 590 & 1.140 & Q & $0,99 *$ & 90 & 770 & 1.360 & 1.300 & $P$ & $0,94 * *$ & 1,1 \\
\hline C. fissilis & 70 & 140 & 250 & 390 & $\mathrm{~L}$ & $0,97 * *$ & 110 & 960 & 870 & 1.090 & $\mathrm{P}$ & $0,96 * *$ & 2,8 \\
\hline C. langsdorffii & 80 & 310 & 320 & 740 & $\mathrm{~L}$ & $0,87^{*}$ & 80 & 740 & 820 & 1.770 & $\mathrm{~L}$ & $0,91 * *$ & 2,4 \\
\hline C. trichotoma & 80 & 600 & 1.100 & 1.320 & $\mathrm{~L}$ & $0,97 * *$ & 50 & 760 & 2.060 & 2.400 & $\mathrm{~L}$ & $0,96 * *$ & 1,9 \\
\hline D. cuneatum & 220 & 400 & 570 & 560 & $\mathrm{~L}$ & $0,84 * *$ & 90 & 2.420 & 1.570 & 1.290 & $P$ & $0,96 * *$ & 2,4 \\
\hline H. courbaril & 60 & 170 & 290 & 350 & $\mathrm{~L}$ & $0,98 * *$ & 20 & 620 & 1.820 & 3.020 & $\mathrm{~L}$ & $0,98 * *$ & 8,5 \\
\hline M. nictidans & 70 & 180 & 920 & 430 & LR & $0,74 * *$ & 80 & 730 & 1.810 & 700 & $P$ & $0,87 * *$ & 1,8 \\
\hline M. caesalpiniaefolia & 100 & 100 & 260 & 330 & $\mathrm{~L}$ & $0,90 *$ & 260 & 830 & 1.840 & 2.370 & $\mathrm{~L}$ & $0,98 * *$ & 7,3 \\
\hline M. peruiferum & 40 & 120 & 570 & 450 & $\mathrm{E}$ & $0,83 * *$ & 40 & 250 & 620 & 530 & $\mathrm{P}$ & $0,86 * *$ & 1,2 \\
\hline M. umbellata & 230 & 100 & 260 & 720 & Q & $0,99 * *$ & 70 & 820 & 1.250 & 560 & Q & $0,96 * *$ & 0,8 \\
\hline P. dubium & 50 & 420 & 610 & 600 & Q & $0,99 *$ & 70 & 1.640 & 3.100 & 2.460 & Q & $0,96 * *$ & 4,1 \\
\hline Piptadenia gonoacantha & 70 & 230 & 610 & 1.330 & $\mathrm{Q}$ & $0,99 * *$ & 50 & 660 & 330 & 1.700 & $\mathrm{P}$ & $0,79 * *$ & 1,3 \\
\hline Platypodium gonoacantha & 90 & 210 & 300 & 510 & $\mathrm{~L}$ & $0,96 * *$ & 70 & 470 & 380 & 1.050 & $\mathrm{~L}$ & $0,80 * *$ & 2,2 \\
\hline S. schottiana & 90 & 180 & 190 & 290 & $\mathrm{E}$ & $0,78 * *$ & 100 & 910 & 1.680 & 1.940 & $\mathrm{~L}$ & $0,96 * *$ & 6,6 \\
\hline S. macranthera & 100 & 320 & 1.180 & 1.160 & $\mathrm{P}$ & $0,90 * *$ & 80 & 720 & 2.810 & 3.220 & $\mathrm{~L}$ & $0,93 * *$ & 2,8 \\
\hline S. granuloso-leprosum & 60 & 620 & 80 & 1.330 & $\mathrm{Q}$ & $0,99 *$ & 60 & 840 & 3.090 & 3.250 & $\mathrm{~L}$ & $0,91 * *$ & 2,5 \\
\hline T. impetiginosa & 290 & 450 & 490 & 370 & n.s. & & 100 & 1.170 & 530 & 1.710 & $\mathrm{P}$ & $0,82 * *$ & 5,0 \\
\hline T. micrantha & 40 & 300 & 710 & 560 & $\mathrm{Q}$ & $0,90 *$ & 110 & 750 & 1.680 & 1.500 & $\mathrm{P}$ & $0,90 * *$ & 2,7 \\
\hline
\end{tabular}

${ }^{1}$ L: regressão linear; Q: regressão quadrática; P: regressão potencial; LR: regressão linear recíproca; E: regressão exponencial.

${ }^{2}$ Relação entre o teor de $\mathrm{Zn}$ na raiz e na parte aérea no nível de $60 \%$ de contaminação.

ns, ${ }^{*} \mathrm{e}{ }^{* *}$ Não-significativo e significativo a $5 \%$ e $1 \%$ de probabilidade, respectivamente. 
encontradas para o teor de $\mathrm{Pb}$, que variou de $\leq 1,0 \mathrm{mg} \mathrm{kg}^{-1}$ a $23,9 \mathrm{mg} \mathrm{kg}^{-1}$ nas espécies mais afetadas: Piptadenia gonoacantha, A. peregrina e $S$. macranthera. M. nictitans e P. dubium retêm proporcionalmente mais o $\mathrm{Pb}$ do que o $\mathrm{Zn}$ e o $\mathrm{Cd}$; portanto, estes últimos são os principais responsáveis pelo efeito fitotóxico nessas espécies.

$\mathrm{O} \mathrm{Zn}$ foi o elemento que exerceu influência sobre o maior número de espécies (Tabela 6), havendo relações inversas entre matéria seca relativa e teor desse elemento em 12 das 20 espécies estudadas. Os níveis críticos de toxidez (NCT = nível que reduz em $10 \%$ a matéria seca relativa) desse elemento foram, em $\mathrm{mg} \mathrm{kg}^{-1}$ de massa seca: S. macranthera $=270 ; C$. trichotoma $=250$; M. umbellata $=210 ; P$. dubium $=150$; T. micrantha $=140 ; M$. caesalpiniaefolia $=110$; A. peregrina e $M$. nictidans $=90 ; C$. fissilis $=80$; Piptadenia gonoacantha $=70$ e $M$. peruiferum $=50$. Portanto, essas espécies apresentaram NCT relati- vamente baixos, em comparação com a faixa considerada por Kabata-Pendias \& Pendias (1984). Como as plantas cresceram em solo contendo níveis elevados de outros metais, pode ter ocorrido efeito fitotóxico aditivo (Kahle, 1993), especialmente com o Cd, que foi também absorvido em grande quantidade. Quanto ao Cd, os níveis críticos de toxidez estimados em 11 espécies ( $S$. macranthera, C. trichotoma, M. umbellata, P. dubium, T. micrantha, M. caesalpiniaefolia, A. peregrina, M. nictidans, $C$. fissilis, Piptadenia gonoacantha $\mathrm{e}$ M. peruiferum) foram inferiores aos valores considerados fitotóxicos por Kabata-Pendias \& Pendias (1984). O NCT de Pb para C. trichotoma, P. dubium e Piptadenia gonoacantha foi menor que $1 \mathrm{mg} \mathrm{kg}^{-1}$, portanto, também abaixo da faixa estabelecida Kabata-Pendias \& Pendias (1984). Como já ressaltado, esses níveis críticos devem ser interpretados com cautela, em face da multicontaminação e das inúmeras interações químicas no solo e físiológicas na plan-

TABELA 4. Médias e regressão do teor de Cd na parte aérea e na raiz e razão Cd na raiz/Cd na parte aérea (R/PA) de mudas de espécies arbóreas, cultivadas em diferentes proporções de solo contaminado com metais pesados.

\begin{tabular}{|c|c|c|c|c|c|c|c|c|c|c|c|c|c|}
\hline \multirow[t]{2}{*}{ Espécie } & \multicolumn{4}{|c|}{$\begin{array}{l}\text { Proporção de solo } \\
\text { contaminado (\%) }\end{array}$} & \multicolumn{2}{|c|}{ Regressão $^{1}$} & \multicolumn{4}{|c|}{$\begin{array}{l}\text { Proporção de solo } \\
\text { contaminado (\%) }\end{array}$} & \multicolumn{2}{|c|}{ Regressão } & \multirow[t]{2}{*}{$\mathrm{R} / \mathrm{PA}^{2}$} \\
\hline & 0 & 20 & 40 & 60 & Mod. & $r^{2}$ & 0 & 20 & 40 & 60 & Mod. & $r^{2}$ & \\
\hline & \multicolumn{4}{|c|}{--- Parte aérea $\left(\mathrm{mg} \mathrm{kg}^{-1}\right)$---- } & & & \multicolumn{4}{|c|}{------- Raiz (mg kg-1) -------- } & & & \\
\hline A. mangium & 0,1 & 2,4 & 7,7 & 14,9 & $\mathrm{~L}$ & $0,95 * *$ & 0,2 & 271,1 & 213,2 & 908,5 & $\mathrm{P}$ & $0,95 * *$ & 62,3 \\
\hline A. peregrina & 0,4 & 1,8 & 6,6 & 19,4 & $\mathrm{E}$ & $0,93 * *$ & 0,5 & 24,6 & 15,9 & 41,7 & $P$ & $0,86 * *$ & 2,3 \\
\hline C. lasiophylla & 0,2 & 1,5 & 13,0 & 20,8 & $\mathrm{~L}$ & $0,93 * *$ & 0,3 & 80,0 & 57,5 & 84,7 & $\mathrm{P}$ & $0,99 * *$ & 4,1 \\
\hline C. fissilis & 0,2 & 4,9 & 6,2 & 9,6 & $\mathrm{~L}$ & $0,95 * *$ & 0,1 & 53,9 & 54,0 & 78,4 & $\mathrm{P}$ & $0,96 * *$ & 8,1 \\
\hline C. langsdorffii & 0,5 & 9,7 & 8,7 & 18,4 & $\mathrm{P}$ & $0,93 * *$ & 0,5 & 46,7 & 42,0 & 89,2 & $\mathrm{P}$ & $0,97 * *$ & 4,9 \\
\hline C. trichotoma & 0,8 & 16,4 & 25,2 & 35,1 & $\mathrm{~L}$ & $0,98 * *$ & 0,1 & 24,6 & 55,4 & 84,2 & $\mathrm{~L}$ & $0,99 * *$ & 2,4 \\
\hline D. cuneatum & 0,8 & 37,0 & 46,2 & 45,5 & Q & $0,99 * *$ & 1,1 & 55,1 & 9,3 & 66,7 & $\mathrm{P}$ & $0,78 * *$ & 1,5 \\
\hline H. courbaril & 0,5 & 3,7 & 5,3 & 6,7 & $\mathrm{P}$ & $0,88 * *$ & 0,0 & 32,4 & 39,1 & 99,8 & $\mathrm{P}$ & $0,98 * *$ & 14,8 \\
\hline M. nictidans & 0,2 & 23,6 & 34,8 & 28,7 & $\mathrm{Q}$ & $0,99 * *$ & 0,3 & 50,8 & 44,5 & 27,4 & Q & $0,93 * *$ & 1,0 \\
\hline M. caesalpiniaefolia & 0,1 & 2,1 & 7,7 & 10,3 & $\mathrm{~L}$ & $0,97 * *$ & 0,2 & 35,5 & 80,1 & 129,6 & $\mathrm{~L}$ & $0,99 * *$ & 12,6 \\
\hline M. peruiferum & 0,2 & 1,9 & 5,6 & 7,9 & $\mathrm{~L}$ & $0,98^{*}$ & 0,3 & 7,2 & 17,4 & 24,7 & $\mathrm{~L}$ & $0,99 *$ & 3,3 \\
\hline M. umbellata & 0,1 & 0,9 & 2,9 & 2,1 & $\mathrm{P}$ & $0,83 * *$ & 0,2 & 51,8 & 30,3 & 14,5 & $\mathrm{P}$ & $0,90 * *$ & 6,8 \\
\hline P. dubium & 0,1 & 13,7 & 19,9 & 25,9 & $\mathrm{~L}$ & $0,95 * *$ & 0,5 & 124,2 & 126,4 & 128,3 & Q & $0,98 * *$ & 5,0 \\
\hline Piptadenia gonoacantha & 0,2 & 3,6 & 17,8 & 47,0 & Q & $0,99 * *$ & 0,8 & 59,6 & 157,1 & 139,3 & $\mathrm{P}$ & $0,97 * *$ & 3,0 \\
\hline Platypodium gonoacantha & 0,6 & 9,2 & 11,6 & 21,6 & $\mathrm{~L}$ & $0,96 * *$ & 1,8 & 18,4 & 11,3 & 34,3 & $\mathrm{P}$ & $0,80 *$ & 1,7 \\
\hline S. schottiana & 0,2 & 11,6 & 8,4 & 14,2 & $\mathrm{P}$ & $0,98 * *$ & 0,1 & 99,9 & 100,1 & 99,6 & $\mathrm{P}$ & $0,99 * *$ & 7,4 \\
\hline S. macranthera & 0,7 & 11,4 & 33,6 & 49,6 & $\mathrm{~L}$ & $0,98 * *$ & 0,5 & 59,1 & 24,9 & 124,3 & $\mathrm{P}$ & $0,84 *$ & 2,6 \\
\hline S. granuloso-leprosum & 0,9 & 32,1 & 32,9 & 64,1 & $\mathrm{P}$ & $0,93 * *$ & 0,5 & 33,0 & 127,6 & 258,7 & Q & $0,99 * *$ & 4,1 \\
\hline T. impetiginosa & 0,5 & 5,1 & 7,6 & 6,6 & $\mathrm{P}$ & $0,92 * *$ & 1,8 & 5,6 & 17,5 & 58,0 & Q & $0,99 *$ & 11,3 \\
\hline T. micrantha & 0,1 & 6,3 & 18,6 & 17,7 & $\mathrm{P}$ & $0,92 * *$ & 0,4 & 50,4 & 62,6 & 60,6 & Q & $0,99 * *$ & 3,6 \\
\hline
\end{tabular}

${ }^{1}$ L: regressão linear; Q: regressão quadrática; P: regressão potencial; E: regressão exponencial.

${ }^{2}$ Relação entre o teor de Cd na raiz e na parte aérea no nível de $60 \%$ de contaminação.

${ }^{*}$ e ${ }^{* *}$ Significativo a $5 \%$ e $1 \%$ de probabilidade, respectivamente. 
ta, a que esses metais estão sujeitos (Barceló \& Poschenrieder, 1992). É interessante o fato de que $A$. mangium foi a única espécie que apresentou relação positiva com o teor de $\mathrm{Cu}$. Já em $A$. peregrina e C. trichotoma houve relação inversa entre matéria seca e os teores de $\mathrm{Cu}$ e Fe. Isto foi também verificado para Mn em C. trichotoma e $S$. macranthera.

Como maneira adicional para avaliar os efeitos da contaminação nos teores dos metais na planta, calculou-se o coeficiente de impacto no teor relativo do metal na raiz e na parte aérea (CITR). Esse índice indica quantas vezes a razão do teor R/PA (raiz/parte aérea) é superior na presença de contaminantes. Valores de CITR maiores que a unidade indicam maior teor proporcional nas raízes da planta no solo contaminado. Este varia, de modo direto, com a capacidade da espécie em limitar a translocação dos elementos da raiz para a parte aérea (Tabela 7). Em relação ao $\mathrm{Zn}$, o CITR variou de 0,59 a 28,30. Verifica-se menor retenção proporcional de $\mathrm{Zn}$ na raiz de $A$. pere- grina e C. lasiophylla em solo contaminado do que no controle. D. cuneatum, A. mangium, M. umbellata, $S$. schottiana, $T$. impetiginosa e $H$. courbaril retiveram grande quantidade de $\mathrm{Zn}$ nas raízes, devendo este ser um mecanismo de proteção dessas espécies ao excesso de metais no solo (Kahle, 1993). O número de espécies que apresentaram CITR para $\mathrm{Cd}$ menor do que uma unidade, foi maior do que o observado no Zn. Várias espécies, como A. mangium, C. langsdorffii, C. trichotoma, H. courbaril, S. macranthera, S. granuloso-leprosum e T. impetiginosa, enquanto que as espécies $P$. dubium, Platypodium gonoacantha, M. nictidans, M. peruiferum, Piptadenia gonoacantha e T. micrantha, retêm pouco desse elemento nas raízes. Considerando esses resultados e diante do fato de que o acúmulo diferencial de metal na raiz é indicativo de tolerância (Baker, 1987), espera-se maior número dessas espécies tolerantes ao Zn que ao Cd. O CITR do $\mathrm{Pb}$ variou de 0,15 em Piptadenia gonoacantha a

TABELA 5. Médias e regressão do teor de $\mathrm{Pb}$ na parte aérea e raízes e razão $\mathrm{Pb}$ na raiz/ $\mathrm{Pb}$ na parte aérea (R/PA) de mudas de espécies arbóreas, cultivadas em diferentes proporções de solo contaminado com metais pesados.

\begin{tabular}{|c|c|c|c|c|c|c|c|c|c|c|c|c|c|}
\hline \multirow[t]{2}{*}{ Espécie } & \multicolumn{4}{|c|}{$\begin{array}{l}\text { Proporção de solo } \\
\text { contaminado }(\%)\end{array}$} & \multicolumn{2}{|c|}{ Regressão $^{1}$} & \multicolumn{4}{|c|}{$\begin{array}{l}\text { Proporção de solo } \\
\text { contaminado (\%) }\end{array}$} & \multicolumn{2}{|c|}{ Regressão } & \multirow[t]{2}{*}{$\mathrm{R} / \mathrm{PA}^{2}$} \\
\hline & 0 & 20 & 40 & 60 & Mod. & $r^{2}$ & 0 & 20 & 40 & 60 & Mod. & $r^{2}$ & \\
\hline & \multicolumn{4}{|c|}{--- Parte aérea $\left(\mathrm{mg} \mathrm{kg}^{-1}\right)$---- } & & & \multicolumn{4}{|c|}{------- Raiz (mg kg-1) ------- } & & & \\
\hline A. mangium & 0,1 & 0,1 & 0,4 & 2,1 & $\mathrm{~L}$ & $0,70^{*}$ & 0,1 & 11,7 & 5,6 & 25,1 & $\mathrm{P}$ & $0,92 * *$ & 12,0 \\
\hline A. peregrina & 0,5 & 0,6 & 0,1 & 7,4 & $\mathrm{Q}$ & $0,91 * *$ & 0,1 & 1,1 & 0,1 & 7,1 & $\mathrm{E}$ & $0,42 *$ & 1,0 \\
\hline C. lasiophylla & 5,5 & 2,8 & 1,3 & 0,1 & $\mathrm{~L}$ & $0,96 * *$ & 1,4 & 2,6 & 2,2 & 2,1 & n.s. & & 16,7 \\
\hline C. fissilis & 0,8 & 1,2 & 0,1 & 1,1 & n.s. & & 0,1 & 2,9 & 0,1 & 2,5 & n.s. & & 2,3 \\
\hline C. langsdorffii & 0,1 & 1,3 & 0,4 & 6,3 & $\mathrm{Q}$ & $0,84 * *$ & 0,1 & 4,0 & 3,9 & 15,1 & $\mathrm{P}$ & $0,90 * *$ & 2,4 \\
\hline C. trichotoma & 0,1 & 1,3 & 2,9 & 6,7 & Q & $0,99 *$ & 0,1 & 1,8 & 5,6 & 14,2 & $\mathrm{~L}$ & $0,90 * *$ & 2,4 \\
\hline D. cuneatum & 0,6 & 2,2 & 1,9 & 1,5 & $\mathrm{P}$ & $0,47 * *$ & 1,0 & 5,5 & 3,9 & 2,5 & $\mathrm{P}$ & $0,55 * *$ & 1,7 \\
\hline H. courbaril & 0,1 & 0,5 & 0,6 & 0,1 & n.s. & & 0,1 & 4,0 & 8,2 & 18,5 & $\mathrm{~L}$ & $0,94 * *$ & 147,0 \\
\hline M. nictidans & 0,1 & 0,8 & 0,8 & 0,1 & n.s. & & 0,6 & 1,8 & 1,7 & 3,4 & $\mathrm{P}$ & $0,54 * *$ & 26,7 \\
\hline M. caesalpiniaefolia & 0,1 & 0,3 & 0,1 & 0,8 & n.s. & & 0,1 & 4,4 & 3,0 & 5,0 & $\mathrm{P}$ & $0,88 * *$ & 7,6 \\
\hline M. peruiferum & 0,1 & 0,5 & 0,4 & 1,3 & $\mathrm{E}$ & $0,44 *$ & 0,1 & 1,1 & 0,9 & 2,5 & $\mathrm{P}$ & $0,61 * *$ & 2,0 \\
\hline M. umbellata & 0,1 & 0,7 & 0,1 & 2,3 & $\mathrm{~L}$ & $0,55^{*}$ & 0,1 & 2,6 & 2,2 & 0,5 & $\mathrm{P}$ & $0,42 *$ & 0,2 \\
\hline P. dubium & 0,1 & 0,1 & 0,4 & 1,2 & $\mathrm{E}$ & $0,62 * *$ & 0,1 & 5,6 & 9,1 & 15,1 & $\mathrm{~L}$ & $0,99 * *$ & 12,8 \\
\hline Piptadenia gonoacantha & 0,1 & 2,7 & 3,9 & 6,4 & $\mathrm{~L}$ & $0,98 * *$ & 0,6 & 1,1 & 2,6 & 3,8 & $\mathrm{E}$ & $0,72 * *$ & 0,6 \\
\hline Platypodium gonoacantha & 0,8 & 2,6 & 0,8 & 1,6 & n.s. & & 1,0 & 3,3 & 0,9 & 5,0 & n.s. & & 4,2 \\
\hline S. schottiana & 0,1 & 0,4 & 0,1 & 1,4 & $\mathrm{E}$ & $0,44^{*}$ & 0,1 & 4,4 & 4,3 & 11,3 & $\mathrm{P}$ & $0,93 * *$ & 7,8 \\
\hline S. macranthera & 0,1 & 0,3 & 0,1 & 0,7 & n.s. & & 0,1 & 1,5 & 6,0 & 6,3 & $\mathrm{~L}$ & $0,89^{*}$ & 23,9 \\
\hline S. granuloso-leprosum & 0,1 & 2,2 & 1,1 & 1,8 & $\mathrm{P}$ & $0,98 * *$ & 0,1 & 2,9 & 3,5 & 6,3 & $\mathrm{P}$ & $0,95 * *$ & 3,5 \\
\hline T. impetiginosa & 2,7 & 17,9 & 3,4 & 6,5 & Q & $0,25 * *$ & 1,4 & 4,4 & 37,5 & 27,7 & $\mathrm{E}$ & $0,81 * *$ & 5,1 \\
\hline T. micrantha & 0,3 & 0,3 & 0,9 & 2,1 & $\mathrm{~L}$ & $0,81^{*}$ & 0,1 & 3,3 & 5,2 & 8,0 & $\mathrm{~L}$ & $0,99^{*}$ & 3,8 \\
\hline
\end{tabular}

${ }^{1}$ L: regressão linear; Q: regressão quadrática; P: regressão potencial; E: regressão exponencial.

${ }^{2}$ Relação entre o teor de $\mathrm{Pb}$ na raiz e na parte aérea no nível de $60 \%$ de contaminação.

ns, ${ }^{*} \mathrm{e}^{* *}$ Não-significativo e significativo a $5 \%$ e $1 \%$ de probabilidade, respectivamente. 
98,00 em H. courbaril. Com exceção de M. umbellata e Piptadenia gonoacantha, todas as espécies foram capazes de reter $\mathrm{Pb}$ nas raízes.

H. courbaril foi a espécie que apresentou maior concentração proporcional de $\mathrm{Zn}$ e $\mathrm{Cd}$ na raiz, em relação à parte aérea. Isto sugere que o mecanismo de tolerância dessa espécie está na sua capacidade de absorver e reter os metais nas raízes, não translocando-os para a parte aérea, evitando danos à planta. Por outro lado, C. fissilis mostrou-se muito pouco afetada pelos metais, chegando a ser estimu- lada pela contaminação para crescimento em altura. No entanto, os valores de CITR para essa espécie foram muito inferiores aos observados para H. courbaril, que foi também pouco afetada pelo excesso de metais no solo. Isto indica que o mecanismo apresentado para $H$. courbaril é diferente daquele existente em $C$. fissilis, que mostrou ser mais limitante na absorção dos metais. Verificou-se que nem todos os metais são igualmente retidos nas raízes das diferentes espécies, o que sugere que a tolerância a um metal não necessariamente garante a
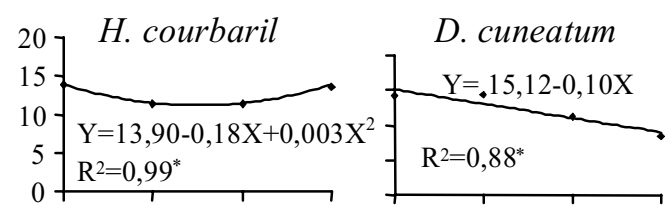

\section{P. dubium}

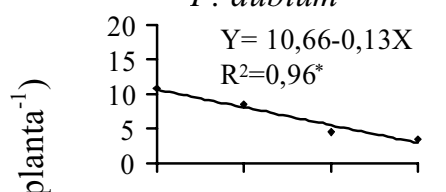

C. fissilis

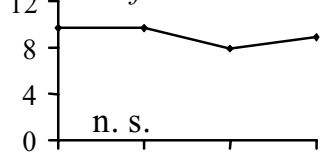

\section{S. macranthera}

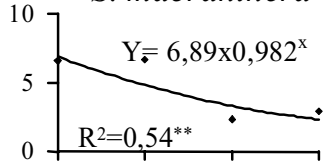

C. langsdorffii

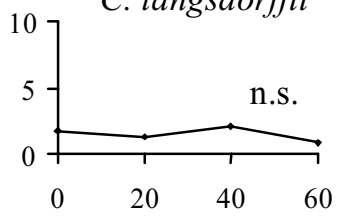

\section{T. micrantha}

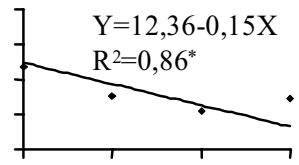

S. granuloso-leprosum

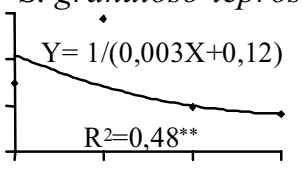

A. mangium

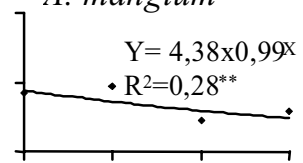

Platypodium

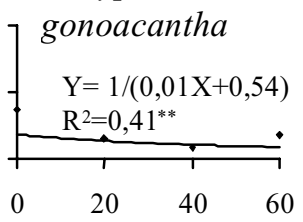

M. umbellata

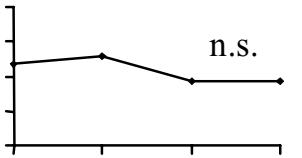

\section{S. schottiana}

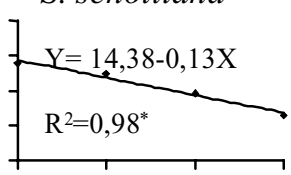

A. peregrina

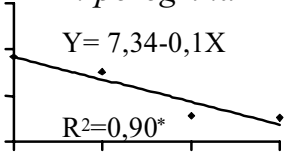

C. trichotoma

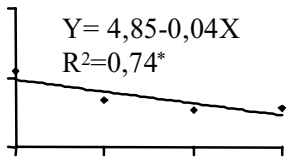

T. impetiginosa

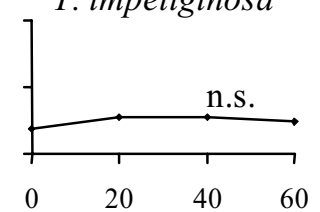

M. caesalpiniaefolia

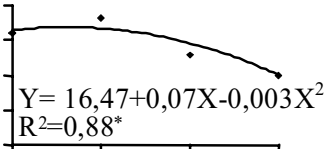

C. lasiophylla

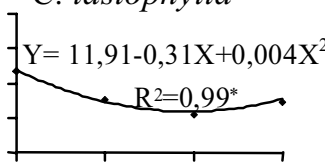

M. nictitans

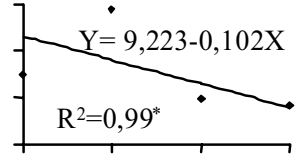

M. peruiferum

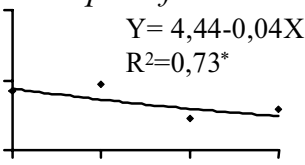

Piptadenia

gonoacantha

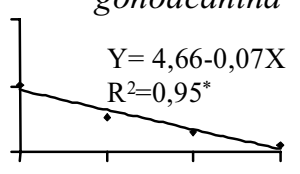

$\begin{array}{llll}0 & 20 & 40 & 60\end{array}$

Níveis de contaminação (\%)

FIG. 2. Respostas de mudas de espécies arbóreas aos tratamentos com diferentes proporções de solo contaminado com metais pesados (n.s: não-significativo; * $\mathbf{p} \leq 0,05 ; * * p \leq 0,01$ ). 
tolerância a outro (Antosiewicz, 1992). A absorção em solo contendo diversos metais pode ser diferente da verificada em elementos isolados, dada as diversas interações entre esses, que podem ser independentes, antagonistas, aditivas ou sinergísticas (Barceló \& Poschenrieder, 1992; Kahle, 1993), e as respostas das espécies ao excesso de metais, diferenciadas em função da especiação desses no solo. McBride (1994) comentou que metais absorvidos na forma catiônica como $\mathrm{Cu}, \mathrm{Pb}$ e $\mathrm{Cd}$, translocam-se pouco para a parte aérea. Entretanto, os resultados aqui apresentados evidenciam uma enorme variação das espécies em relação à capacidade de absorver e translocar o $\mathrm{Zn}, \mathrm{Cd}$ e $\mathrm{Pb}$. De modo geral, verifica-se que as espécies menos afetadas pela contaminação concentram os metais absorvidos nas raízes, portanto limitando sua translocação para a parte aérea. Isto resulta de mecanismos diversos que reduzem a disponibilidade na rizosfera, a retenção dos metais na parede celular ou na superfície da raiz e a imobilização no citoplasma (Kahle, 1993), diminuindo sua atividade na planta.

A tolerância das plantas aos metais pesados tem sido bem documentada em espécies herbáceas (Brooks et al., 1981), e raramente nas espécies arbóreas (Kahle, 1993). Mesmo sem evidências de existência de caracteres genéticos para tolerância (Wilkinson et al., 1992; Wilkinson \& Dickinson, 1995),

TABELA 6. Equações de regressão da massa de matéria seca relativa e teores dos metais pesados na parte aérea. Espécies com ajustes significativos e $R^{2} \geq 0,65$.

\begin{tabular}{|c|c|c|c|c|}
\hline Espécie & Equação & $\mathrm{R}^{2}$ & $\mathrm{P} \leq$ & Metal \\
\hline A. mangium & $Y=25,7874+2061,36 X-12846,2 X^{2}$ & 0,79 & 0,01 & $\mathrm{Cu}$ \\
\hline \multirow{3}{*}{ A. peregrina } & $Y=114,422-293,741 X-229,258 X^{2}$ & 0,90 & 0,01 & $\mathrm{Zn}$ \\
\hline & $\mathrm{Y}=80,9407-19,39391 \mathrm{nX}$ & 0,86 & 0,01 & $\mathrm{Cd}$ \\
\hline & $\mathrm{Y}=3,49516+1,4102 \mathrm{X}^{-1}$ & 0,84 & 0,01 & $\mathrm{Cu}$ \\
\hline C. lasiophylla & $Y=96,2115-13,114 X+0,550373 X^{2}$ & 0,96 & 0,01 & $\mathrm{Cd}$ \\
\hline \multirow{2}{*}{ C. fissilis } & $Y=99,0023-142,165 X+368,836 X^{2}$ & 0,88 & 0,01 & $\mathrm{Zn}$ \\
\hline & $\mathrm{Y}=92,0929-2,90476 \mathrm{X}+0,354589 \mathrm{X}^{2}$ & 0,81 & 0,01 & $\mathrm{Cd}$ \\
\hline \multirow[t]{4}{*}{ C. trichotoma } & $Y=107,861-78,7313 X+25,3051 X^{2}$ & 0,96 & 0,01 & $\mathrm{Zn}$ \\
\hline & $Y=103,701-20,6093 X+3,692 X^{2}$ & 0,91 & 0,01 & $\mathrm{Cd}$ \\
\hline & $\mathrm{Y}=53,1004+6,38927 \mathrm{X}^{-1}$ & 0,75 & 0,01 & $\mathrm{~Pb}$ \\
\hline & $\mathrm{Y}=30,7202+2,00779 \mathrm{X}^{-1}$ & 0,65 & 0,01 & $\mathrm{Cu}$ \\
\hline M. nictidans & $\mathrm{Y}=54,2826+3,36331 \mathrm{X}^{-1}$ & 0,68 & 0,01 & $\mathrm{Zn}$ \\
\hline \multirow[t]{2}{*}{ M. caesalpiniaefolia } & $Y=147,182-651,565 X+1202,52 X^{2}$ & 0,88 & 0,01 & $\mathrm{Zn}$ \\
\hline & $Y=94,766-3,33615 X$ & 0,69 & 0,05 & $\mathrm{Cd}$ \\
\hline \multirow[t]{2}{*}{ M. peruiferum } & $\mathrm{Y}=29,5883+2,91093 \mathrm{X}^{-1}$ & 0,99 & 0,01 & $\mathrm{Zn}$ \\
\hline & $Y=114,661-61,4577 X^{0,5}+11,748 X$ & 0,93 & 0,01 & $\mathrm{Cd}$ \\
\hline \multirow[t]{2}{*}{ M. umbellata } & $Y=103,281-68,5493 X+29,3298 X^{2}$ & 0,67 & 0,01 & $\mathrm{Zn}$ \\
\hline & $Y=103,701-20,6093 X+3,692 X^{2}$ & 0,72 & 0,01 & $\mathrm{Cd}$ \\
\hline \multirow[t]{3}{*}{ P. dubium } & $Y=(0,006029+0,0337888 X)^{-1}$ & 0,91 & 0,01 & $\mathrm{Zn}$ \\
\hline & $Y=100,427-1,29833 X-0,0466657 X^{2}$ & 0,91 & 0,01 & $\mathrm{Cd}$ \\
\hline & $Y=(0,0110816+0,0158339 X)^{-1}$ & 0,66 & 0,01 & $\mathrm{~Pb}$ \\
\hline \multirow[t]{4}{*}{ Piptadenia gonoacantha } & $\mathrm{Y}=10^{(2,00776-0,762404 \mathrm{X})}$ & 0,98 & 0,01 & $\mathrm{Zn}$ \\
\hline & $Y=90,4556-4,28743 X+0,054962 X^{2}$ & 0,93 & 0,01 & $\mathrm{Cd}$ \\
\hline & $Y=102,839-17,9787 X+0,533168 X^{2}$ & 0,97 & 0,01 & $\mathrm{~Pb}$ \\
\hline & $Y=-16,9279+10279,7 X-197126 X^{2}$ & 0,88 & 0,01 & $\mathrm{Cu}$ \\
\hline \multirow[t]{3}{*}{ S. macranthera } & $\mathrm{Y}=107,489-69,4995 \mathrm{X}+15,9813 \mathrm{X}^{2}$ & 0,86 & 0,01 & $\mathrm{Zn}$ \\
\hline & $Y=102,9-2,09664 X+0,018923 X^{2}$ & 0,75 & 0,01 & $\mathrm{Cd}$ \\
\hline & $\mathrm{Y}=155,908-3300,12 \mathrm{X}+2243,51 \mathrm{X}^{2}$ & 0,90 & 0,01 & $\mathrm{Mn}$ \\
\hline \multirow[t]{2}{*}{ S. granuloso-leprosum } & $\mathrm{Y}=5,32961+252,414 \mathrm{X}^{0,5}-189,962 \mathrm{X}$ & 0,73 & 0,01 & $\mathrm{Zn}$ \\
\hline & $Y=-108,776+1784,46 X^{0,5}-4099,15 X$ & 0,65 & 0,01 & $\mathrm{Mn}$ \\
\hline \multirow[t]{2}{*}{ T. micrantha } & $Y=107,828-139,206 X+76,0568 X^{2}$ & 0,93 & 0,01 & $\mathrm{Zn}$ \\
\hline & $Y=101,624-4,16674 X+0,0712878 X^{2}$ & 0,98 & 0,01 & $\mathrm{Cd}$ \\
\hline
\end{tabular}


TABELA 7. Coeficiente de impacto no teor proporcional (CITR) de metal entre a raiz e a parte aérea (R/PA) das espécies.

\begin{tabular}{|c|c|c|c|}
\hline Espécies & $\mathrm{Zn}$ & $\mathrm{Cd}$ & $\mathrm{Pb}$ \\
\hline & \multicolumn{3}{|c|}{ - } \\
\hline A. mangium & 5,71 & 14,16 & 12,00 \\
\hline A. peregrina & 0,59 & 2,09 & 1,43 \\
\hline C. lasiophylla & 0,69 & 2,73 & 55,67 \\
\hline C. fissilis & 1,87 & 2,38 & 5,75 \\
\hline C. langsdorffii & 2,40 & 5,44 & 2,40 \\
\hline C. trichotoma & 3,17 & 24,00 & 2,40 \\
\hline D. cuneatum & 6,00 & 1,07 & 1,70 \\
\hline H. courbaril & 28,30 & 148,00 & 98,00 \\
\hline M. nictidans & 1,64 & 0,56 & 26,70 \\
\hline M. caesalpiniaefolia & 2,70 & 1,80 & 1,90 \\
\hline M. peruiferum & 1,33 & 0,81 & 2,00 \\
\hline M. umbellata & 4,00 & 1,02 & 0,20 \\
\hline P. dubium & 2,93 & 0,43 & 12,80 \\
\hline Piptadenia gonoacantha & 1,63 & 0,23 & 0,15 \\
\hline Platypodium gonoacantha & 2,80 & 0,50 & 2,47 \\
\hline S. schottiana & 5,50 & 2,55 & 7,80 \\
\hline S. macranthera & 3,50 & 3,71 & 23,90 \\
\hline S. granuloso-leprosum & 2,50 & 8,20 & 3,50 \\
\hline T. impetiginosa & 16,67 & 2,90 & 1,38 \\
\hline T. micrantha & 1,04 & 0,49 & 5,43 \\
\hline
\end{tabular}

${ }^{1} \mathrm{CITR}=$ razão do teor R/PA de cada elemento no tratamento com $60 \%$ de contaminação dividido pela razão do teor R/PA no controle sem contaminação

algumas espécies de árvores podem sobreviver em condições de excesso de metais no solo, como indicam os resultados deste trabalho, embora por um curto período estudado. Segundo Dickinson et al. (1992), a plasticidade fenotípica pode permitir a sobrevivência em condições adversas, após algum período de aclimatação à toxidez de metal. De fato, em áreas com elevado grau de contaminação, verificase que algumas espécies arbóreas toleram o estresse do excesso de metais no solo, como é o caso da cagaita - Eugenia dysenterica - e do ingá-bravodo-cerrado - Inga sp. - encontradas na área onde foi coletado o solo contaminado utilizado no presente estudo.

\section{CONCLUSÕES}

1. As espécies arbóreas se comportam de modo diferenciado frente à contaminação do solo com metais pesados.
2. C. fissilis, M. umbellata, T. impetiginosa, C. langsdorffii, H. courbaril, M. caesalpiniaefolia, A. mangium e Platypodium gonoacantha são as espécies menos afetadas, destacando-se $C$. fissilis, que apresenta elevada tolerância ao excesso de metais pesados no solo.

3. A resposta da maioria das espécies à contaminação do solo com metais pesados relaciona-se à capacidade destas de acumular $\mathrm{Zn}$ e $\mathrm{Cd}$ nas raízes ou de translocá-los para a parte aérea.

\section{REFERÊNCIAS}

ANTOSIEWICZ, D.M. Adaptation of plants to an environment polluted with heavy metals. Acta Societatis Botanicorum Poloniae, v.61, n.2, p.281299, 1992.

BAKER, A.J.M. Accumulators and excluders - strategies in the response of plants to heavy metals. Journal

Pesq. agropec. bras., Brasília, v.35, n.1, p.121-132, jan. 2000 
of Plant Nutrition, New York, v.3, n.1/4, p.643654, 1981.

BAKER, A.J.M. Metal tolerance. The New Phytologist, London, v.106, p.93-111, 1987.

BARCELÓ, J.; POSCHENRIEDER, C. Respuestas de las plantas a la contaminación por metales pesados. Suelo y Planta, v.2, n.2, p.345-361, 1992.

BROOKS, R.R.; SHAW, S.; MARFIL, A.A. The chemical form and physiological function of nickel in some Iberian Alyssum species. Physiologia Plantarum, v.51, p.167-170, 1981.

BROWN, S.L.; CHANEY, R.L.; ANGLE, J.S.; BAKER, A.J.M. Zinc and cadmium uptake by hyperaccumulator Thlaspi caerulescens grown in nutrient solution. Soil Science Society of America. Journal, Madison, v.59, n.1, p.125-133, 1995.

DICKINSON, N.M.; TURNER, A.P.; WATMOUGH, S.A.; LEPP, N.W. Acclimation of trees to pollution stress: cellular metal tolerance traits. Annual Botany, v.70, p.569-572, 1992.

EMBRAPA. Serviço Nacional de Levantamento e Conservação de Solos (Rio de Janeiro, RJ). Manual de métodos de análise de solo. Rio de Janeiro, 1979. Não paginado.

FOSTER, J.C. Total heavy metal contents by aqua regia digestion. In: ALEF, K.; NANNIPIERI, P. (Eds.) Methods in applied soil microbiology and biochemistry. San Diego : Academic, 1995. p.101102.

KABATA-PENDIAS, A.; PENDIAS, H. Trace elements in soils and plants. Boca Raton : CRC, 1984. 315p.
KAHLE, H. Response of roots of trees to heavy metals. Environmental and Experimental Botany, v.33, n.1, p.99-119, 1993.

McBRIDE, M.B. Environmental chemistry of soil. New York : Oxford Univ. Press, 1994. 406p.

SALT, D.E.; BLAYLOCK, M.; KUMAR, N.P.B.A.; DUSHENKOV, V.; ENSLEY, B.D.; CHET, I.; RASKIN, I. Phytoremediation: A novel strategy for the removal of toxic metals from the environment using plants. Biotechnology, v.13, p.468-474, 1995.

UNIVERSIDADE FEDERAL DE VIÇOSA. Sistema para análise estatística (SAEG): guia de uso resumido. Viçosa : Fundação Arthur Bernardes, Divisão de Informática, [19--].

VERKLEIJ, J.A.C.; PRAST, J.E. Cadmium tolerance and co-tolerance in Silene vulgaris (Moench.) Garcke [= S. cucubalus (L.) wilb.]. The New Phytologist, London, v.111, n.4, p.637-645, 1989.

WILKINSON, D.M.; DICKINSON, N.M. Metal resistance in trees: the role of mycorrhizae. Oikos, v.72, n.2, p.298-300, 1995.

WILKINSON, D.M.; TURNER, A.P.; WATMOUGH, S.A.; LEPP, N.W. Acclimation of trees to pollution stress: cellular metal tolerance traits. Annual Botany, v.70, p.569-572, 1992.

ZONTA, E.P.; MACHADO, A.A.; SILVEIRA JÚNIOR, P. Sistemas de Análise Estatística para Microcomputadores (SANEST). Pelotas : UFPel, Dep. de Matemática e Estatística, 1984. 151p. 